\title{
The method of bimoment distribution for the analysis of continuous thin-walled structures subject to torsion
}

\author{
A. H. KHAN \& H. TOTTENHAM
}

\section{Dr G. H. Béguin, Geneva}

The Paper presents designers with a useful hand method for analysing warping problems in thin-walled members subjected to torsion.

38. To the two factors mentioned in $\S 1$ as contributing to the growing importance of torsion, should be added the steady decrease in wall thickness which is accompanying the increasing use of structural members made of metal strips. The line element is still the basic element of steel construction; the replacement of the line element by sheet element needs further study. ${ }^{\theta}$ The only sheet elements which are used to a certain extent as two-dimensional load-carrying elements are the orthotropic deck panel and some types of metal panel stiffened by impressed deformations, as used in the container and automobile industries. Corrugated sheet can hardly qualify as a two-dimensional load carrying element, because its stiffness along the corrugation can be some hundred times greater than in the perpendicular direction.

39. In Table $1(\$ 14)$ the Authors present expressions for bimoments in a single span beam subject to various loadings and end conditions. In an investigation on using prefabricated deck sections in a new method of deck construction for composite bridges, I had to compute the cross-section twist $\theta(z)$ of continuous steel beams of open crosssection-a common section in composite bridges. First, for a single span simply supported at both extremities, and with both end sections free to warp $\left(\theta^{\prime} \neq 0\right)$ but not to twist $(\theta=0)$, I derived expressions

(a) for the warping $\theta^{\prime}(0)$ and $\theta^{\prime}(l)$ at the beam extremities

(b) for the unit transverse (weight) component $S$, defined as

$$
S=\int_{0}^{l} \theta(z) \mathrm{d} z \text {. }
$$

40. The expressions were derived for several common loading cases. For ease of computation and for rapid determination of the order of magnitude of the displacements and forces, series expansions in ascending powers of $k l$ were also derived for the case of open cross-section $(k l<1){ }^{\theta}$ To enhance the value of the results given by the Authors, the expressions in Table 1 could also easily be expanded in power series with ascending powers of $k l$ or $1 / k l$, depending on the magnitude of $k l$. The Authors' expressions often consist of the subtraction of terms nearly equal in magnitude so that the order of magnitude of the result, and thus the significance of the computed bimoments, are not readily perceived. 
41. For the thin-walled continuous beam, an equation can easily be established which is similar to the three moments equation of classical continuous (solid) beam theory, which for the case $\mu=1$ is

$$
B_{j} C_{j}(k l)-B_{j+1} D_{j}(k l)-B_{j+1} D_{j+1}(k l)+B_{j+2} C_{j+1}(k l)=L(P, k l) .
$$

where $B$, is the unknown bimoment in the section over the $j$ th support. The terms $C_{j}$ and $D_{j}$ pertain to the span running between the $j$ th and the $(j+1)$ th support and are

$$
\begin{aligned}
& C_{f}=\frac{1}{G J_{\mathrm{d}} l} \frac{k l-\sinh k l}{\sinh k l} \\
& D_{f}=\frac{1}{G J_{\mathrm{d}} l} \frac{(k l) \cosh k l-\sinh k l}{\sinh k l}
\end{aligned}
$$

or

$$
\left.\begin{array}{l}
C_{f}=\frac{1}{G J_{\mathrm{d}} l} c(k l) \\
D_{j}=\frac{1}{G J_{\mathrm{d}} l} d(k l)
\end{array}\right\} .
$$

The term $L(P, k l)$ is the loading term.

42. The series expansions for $c(k l)$ and $d(k l)$ are

$$
\begin{aligned}
& c(x)=-x^{2}\left[\frac{1}{6}-\frac{7 x^{2}}{360}+\frac{31 x^{4}}{21 \times 720}+\ldots\right] . \quad . \quad . \\
& d(x)=x^{2}\left[\frac{1}{3}-\frac{x^{2}}{45}+\frac{2 x^{4}}{21 \times 45}+\ldots\right] . . .
\end{aligned}
$$

where $x$ stands for $(k l)$.

43. For a continuous thin-walled beam with $n$ equal spans, a constant cross-section, and for which the twist of the cross-section is prevented over each support, a scheme based on a second order linear difference equation easily yields an expression for the bimoment $B_{f}$ at each support for two cases: equally loaded spans and a beam subjected only to a concentrated bimoment $B$ acting at one end support. For the latter case, the bimoment $B$, is given by

$$
B_{j}=(-1)^{n+1} B \frac{\sinh j \gamma}{\sinh n \gamma} \quad . \quad . \quad . \quad . \quad .
$$

where

$$
\begin{aligned}
j & =1,2, \ldots \\
\cosh \gamma & =\frac{(k l) \cosh k l-\sinh k l}{\sinh k l-k l}
\end{aligned}
$$

44. For the warping of the terminal section (the one at which the bimoment $B$ is applied) the following approximation ${ }^{10}$ is justified whenever $n>3$ and the cross-section is open $(k l \ll 1)$

$$
\theta_{n}^{\prime}=\frac{B}{G J_{\mathrm{d}} l}(k l)^{2} \sqrt{ } 3\left[\frac{1}{6}-\frac{(k l)^{2}}{120}+\ldots\right] \quad . \quad . \quad . \quad .
$$

45. My work is also based on Vlasov's theory of thin-walled beams ${ }^{1}$ and the corresponding assumptions - it is one of the most useful theories available.

\section{Dr Khan and Mr Tottenham}

On page 862 of the Paper, equation (6) should read

$$
\mathbf{Z}_{b}=\mathbf{G}(b) \mathbf{Z}_{a} \text {, right }
$$


47. Dr Béguin draws attention to the fact that the increasing use of thin metal sections in modern steel constructions has emphasized the need for torsion analysis of structures in their design. We believe this to be true, and because such sections are likely to have local buckling as their failure criteria, this makes it imperative for the designers to examine the effect of torsion in their overall stress distributions.

48. He suggests that the various expressions for the fixed end bimoments should be given as a power series for the characteristic torsion parameter $k l$. Although the resulting numerical values for some of these expressions may be small compared with the individual terms, it is unlikely that any significant error will be introduced if tables of hyperbolic functions are used. The value of $k l<0.5$ is very unusual and in this case the round-off error will be less than $1 \%$. We give the expressions in the form of hyperbolic functions as these are readily calculated on most scientific calculators and give an accuracy which is more than sufficient, even for the smallest practical value of $\mathrm{kl}$.

49. We agree that a 'three bimoment' equation can be set up. The object of the distribution procedure outlined was to avoid the need to solve such equations.

50. The analysis presented, when used for open sections, is based on the well-known Vlasov theory. This theory has been subjected to many tests and found to predict well the behaviour of thin-walled beams of open profile. The analysis may also be used in the design of continuous box beams or beams having a mixed open/closed profile. However, there is insufficient experimental evidence to confirm the exactness of this analysis for these types of member. Apart from regions under loads where deformation of the cross-section may occur, the basic assumptions appear to be as fully satisfied as they are for open profiles.

\section{References}

8. Béguin G. H. and Trudel F. Ist die Anwendung von Stahlblech im Wohnungsund Hochbau gerechtfertigt? (Is the use of steel sheet structural members in dwellings and buildings justified?) Blech, 1975, 22, May, 216-221.

9. BÉGUIN G. H. The steering of a concrete deck on the flanges of steel girders. Struct. Engr, 1978, 56B, No. 1, Mar., 7.

10. BéGUIN G. H. Decking of composite bridges by stage-deck-jacking. J. Struct. Div. Am. Soc. Civ. Engrs, 1978, 104, ST 1, Jan., 171-189. 